\title{
European and Swiss Congress of Internal Medicine
}

\author{
Geneva, May 14-16, 2014
}

The $13^{\text {th }}$ Congress of the European Federation of Internal Medicine (EFIM) was a joint project of the Swiss Society of General Internal Medicine (SSGIM) and the EFIM. It was a common venture of the EFIM Annual Congress and the $82^{\text {nd }}$ Annual Meeting of the SSGIM. The idea of incorporating the two congresses reflects the strong tradition of internal medicine in Switzerland and aimed to combine lectures of internal medicine generalists with specialists as well as reflecting the shift from medical care to interdisciplinary care. A number of Swiss medical societies (e.g. Hematology Society, Clinical Pharmacology and Toxicology Society, Clinical Pharmacology and Toxicology Society, Hypertension Society, Physical Medicine and Rehabilitation Society, Academy for Psychosomatic and Psychosocial Medicine and a few others) were invited to be co-organizers of the congress. The post of Congress President was taken by Professor Arnaud Perrier. The Congress was very successful. It was attended by 3700 participants from all over the world (57 countries), and included 90 workshops, 25 invited societies sessions, 14 symposia and 10 meet-the-experts sessions. The meeting took place in the famous convention center Palexpo.

The first point of the Opening Ceremony of the Congress was a welcoming address by Arnaud Perrier, the President of the Congress. On behalf of the Swiss Society of General Internal Medicine, the participants were welcomed by Jean-Michel Gaspoz and on behalf of the EFIM by Maria Domenica Cappellini. The welcome address on behalf of the Foundation for Development of Internal Medicine in Europe was delivered by Daniel Sereni.

The artistic break featured an excellent live performance by Laura von Bongard and Pavel Stankevych and was followed by the opening lecture: "Do quality efforts improve patients' outcomes?" (David Bates). The abstract session entitled "Infections diseases, immunology and rheumatology" contained 225 contributions.

The rheumatological main session was chaired by Eugeniusz J. Kucharz, and the lecture "Should we treat osteoarthritis aggressively?" was delivered by Pascal Daniel Zufferey from Lausanne. A poster presentation from Poland entitled "Satisfaction and dissatisfaction of patients with rheumatic diseases treated with biologics" by Anna Kotulska, Piotr Wiland, Eugeniusz J. Kucharz et al. should also be mentioned.

The other topics related to rheumatology included "Update - bone diseases" (Michael Brändle, Francesca Mainieri - chairpersons, Brigitte Übelhart - Bone diseases, Nicolas Rodonti - Preventive medicine and bone). Valérie Pignet delivered a lecture entitled "How to manage osteoarticular pain". Some communications were

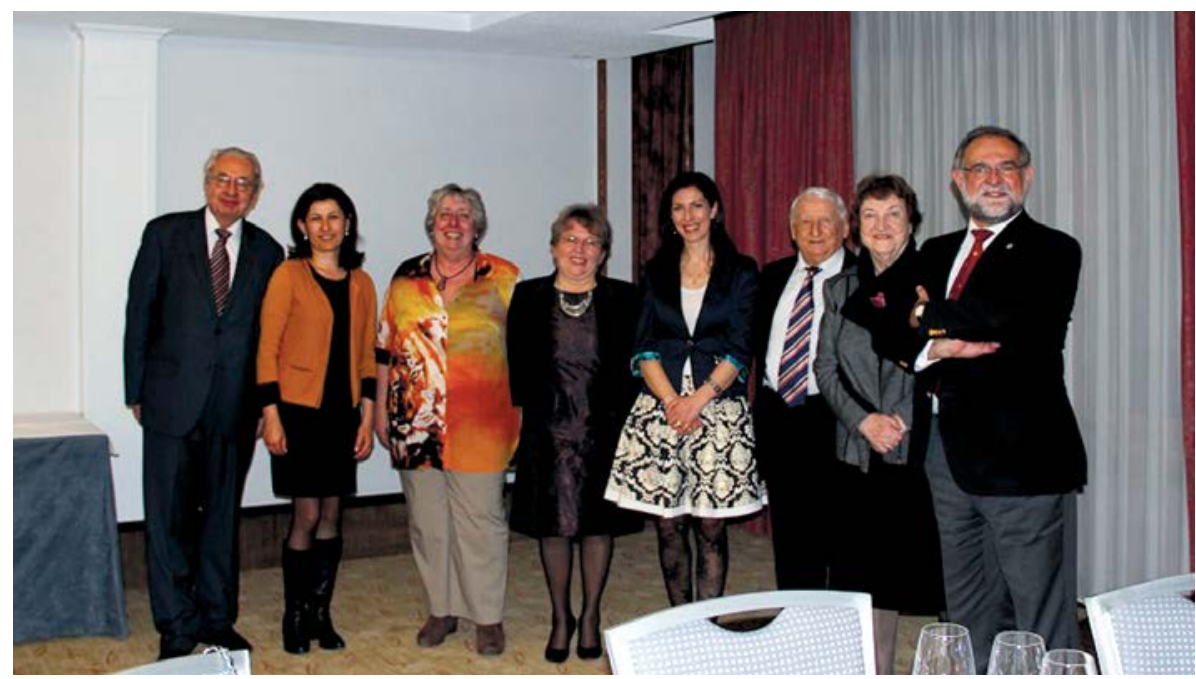

Photo 1. Geneva, May 15, 2014. During the welcome dinner. From left: Eugeniusz J. Kucharz, NN, Karena Grundy, Anna Kotulska, Aneta Trajkovska, Primož Vidali, Jilka Vidali, Faustino Ferreira. 


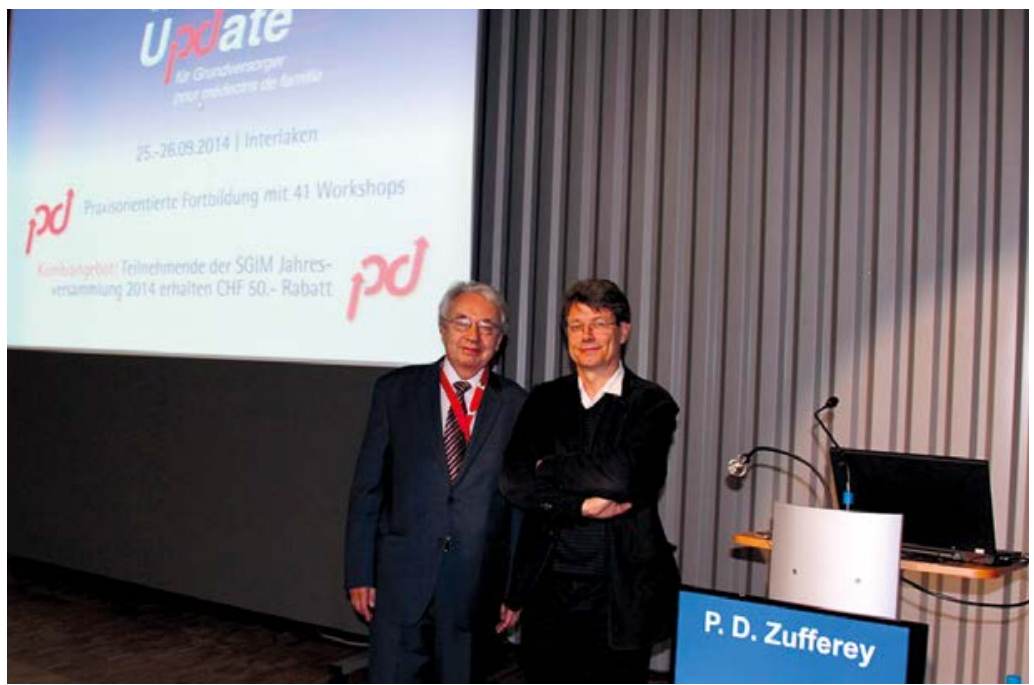

Photo2.Geneva,May17,2014.Rheumatologicalsession.Fromleft:Eugeniusz J. Kucharz and Pascal Daniel Zufferey.

accepted for oral presentation and the session was chaired by Daniel Sereni and Jean Dudler. The papers' titles are: "The lumbar spine trabecular bone score" (Olivier Lamy), "Interdependence between pain, functional disability and disease activity in psoriatic arthritis patients with axial involvement" (Elena Cerlat), "Creating a miscarriage risk score in mothers with autoimmune diseases" (Javier Moreno Diaz), and "Level of agreement in Tunisian physicians ACR-EULAR 2013 recommendations in management of rheumatoid arthritis" (Kaouther Maatallah). Two practical training courses on rheumatologic examination were provided by Jean Dufler in French and in English.

A very special event was the Raths-Steiger Lecture. The session was chaired by Rolf Streuli, and the lecture "Allocating scarce medical resources" was delivered by Ezekiel J. Emanuel from the American College of Physicians. It was an excellent presentation of various aspects of the health system reform in the United States of America, presented in comparison with other countries. The lecture was named after Marguerite Steiger (1908-1990) and Hermine Raths (1906-2005), Swiss researchers in chemistry and pharmacology.

During the Congress the meeting of the EFIM Administrative Council took place. Poland was represented at the Council by Anna Kotulska and Eugeniusz J. Kucharz. At the meeting, a number of issues were discussed. The president of the EFIM, Maria Domenica Cappellini, reported contacts with the European Union authorities and the American College of Physicians. Lorenco Dagna and Maria Domenica Cappellini had participated in the meeting of the Expert Group on Rare Diseases in Luxembourg. The new website of the EFIM was presented. A report from the joint meeting of the Union Européenne des Médecins Spécialistes - European Board of Internal Medicine and the EFIM was delivered.

During the Congress a meeting of the Editorial Board of the European Journal of Internal Medicine took place. Eugeniusz J. Kucharz is a member of the International Committee of the journal. A 20\% increase in manuscript submission compared to 2013 was noted. The journal is affiliated with the Polish Society of Internal Medicine.

The Cyprus Society of Internal Medicine became a member of the EFIM. The Society was founded in 1980, and currently has 70 active members and 60 old members. At the meeting the Society was represented by Irene Kaoulla Gase. The Society will host the EFIM in 2015.

The European and Swiss Congress of Internal Medicine was a very successful meeting, both as a scientific and an educational event.

Eugeniusz J. Kucharz 